\title{
Haptic Feedback Experiments for Improved Teleoperation of a Robotic Arm
}

\author{
Monica Dascalu ${ }^{1,4}$, Lucian Milea ${ }^{2}$, Eduard Franti ${ }^{1,3}$, Florin Lazo ${ }^{1}$, Elteto Zoltan ${ }^{1}$, Ioan Ispas ${ }^{1}$ \\ ${ }^{1}$ Research Institute for Artificial Intelligence, Department for New Electronic Architecture, Bucharest, Romania \\ ${ }^{2}$ Solaris Consult, Bucharest, Romania \\ ${ }^{3}$ National Institute for Research and Development in Microtechnologies, Department, Modelling and Computer-Aided Design Laboratory, \\ Bucharest, Romania \\ ${ }^{4}$ Politehnica University of Bucharest, Faculty of Electronics, Telecommunication and Information Technology, Department of Electronic \\ Architectures, Devices and Circuits, Bucharest, Romania
}

\section{Email address:}

monicad@artsoc.ro (M. Dascalu), lucian@artsoc.ro (L. Milea), edif@artsoc.ro (E. Franti), florin_lazo@yahoo.com (F. Lazo), zelteto@gmail.com (Z. Elteto),john_17_08@yahoo.com (I. Ispas)

\section{To cite this article:}

Monica Dascalu, Lucian Milea, Eduard Franti, Florin Lazo, Elteto Zoltan, Ioan Ispas. Haptic Feedback Experiments for Improved Teleoperation of a Robotic Arm. American Journal of Aerospace Engineering. Vol. 3, No. 3, 2016, pp. 36-42.

doi: 10.11648/j.ajae.20160303.13

Received: October 31, 2016; Accepted: November 9, 2016; Published: December 5, 2016

\begin{abstract}
The paper presents a robotic arm which is operated by means of a sensorial interface mounted on the hand and arm of the human operator. The novelty of the research is the application of devices similar to those used for movements detection in virtual reality applications in order to command a robotic system. Depending on the precision of the application intended and also depending on the number of degrees of freedom, the motion detection for the human hand and arm's motions was approached on different levels of complexity. The data processing methods and the action commands methods were developed in correlation with the structure of the robotic arm and starting from the monitor of the movements of the human arm and hand. The sensorial interface was conceived on the premises that the robotic arm should be able to realize movements similar to those of a healthy human hand, as requested by the application. Therefore, the sensorial interface that monitors the movement of the hand was implemented in order to command a robotic arm having 5 degree of freedom, having an anthropomorphic robotic hand at its end The joints of the system allow rotations of 30-180 degrees (depending on the utility and position). The experimental testing of the robotic system verified the performances of the robotic arm to replicate the movements of the human hand. The operator executed a sequence of movements, with the sensorial interface on, and the robotic arm reproduced the movements (the response was analyzed in quality and quantity).
\end{abstract}

Keywords: Robotic Arm, Haptic Feedback, Teleoperation

\section{Introduction}

In the last years, different groups of researchers and several companies have designed a large variety of anthropomorphic mechanical arms with ever growing performances. Some of the products available now have a flexibility comparable to that of a healthy human hand, which allows a large variety of handling operations for different types of objects. However, there is still more research necessary in order to conceive and develop the algorithms that make possible specific sets of complex movements for such anthropomorphic robotic arms.

The methods most frequently used for remote control of the robotic arms include joysticks, preset sets of commands or the replication of the movements of a human operators's arm. The last of these methods is the most efficient, regarding the performance-cost ratio. The method implies the use of several devices: electromagnetic sensors, movement sensors, bending sensors, gloves with bending and pressure sensors and accelerometers etc. It can be used both with 
wired command devices or with wireless connection to the command unit.

A significant limitation of the command systems of the teleoperate robotic arms is established by the feedback provided by the dedicated circuits implemented on the robotic arm. The feedback can provide a survey mechanism for the operator, in order to look after the operations realized by the robotic arm. This is one of the main reasons why almost all most performant robotic arms realized in the recent period implement several artificial intelligence features or functions, being able to make decisions regarding, for instance, the force of the ...or the speed of the movement, in order not to break the objects manipulated or the objects in the surrounding environment [1]. For such functions, the command and control block may require a microprocessors network, each dedicated to one specific action. Excepting the full autonomous robots, the implementation of one or several feedback levels is indispensable in order to efficiently command the movements (of a tele-operated robotic arm). [2]

Without several types of sensors, it wouldn't be possible to duplicate the movements of the human hand. The applications that require remote control of a robotic arm belong to the category of operations in dangerous environments and remote or inaccessible (for the human operator) environments - in such situations a solution frequently used is to duplicate the movements of the hands/arms of a human operator. In some situations, it is cheaper and more efficient to program and teleoperate a robot that is placed in such a medium than to have a risky and/or very expensive human operator intervention. In addition, due to the advances and technological development of the robot and automation industry, nowadays the performances and abilities of the robotic arms may overpass the human abilities and performances (in terms of strength, precision, reproducibility etc.) Such abilities, combined with adequate sensorial systems and intelligent data processing will allow a perfect control of the robotic systems that may function, when necessary, as efficient and safe extensions of the human locomotory system. There are several fields and applications where already robots are considered more efficient than humans and the domain of these application is constantly extending, as it is much more adequate to use specialized robots than human operators in situations where robots can efficiently realize complex operations in handling objects of different sizes and weights with high precision. While several robots are program to repeat specific operations, other robotic arms are controlled by the human operators with specific devices that allow the remote control: joysticks, spacebars, motion detection (and duplication of the human movements). Of all those types of commands, the last requires most information processing: the sensors are placed on the joints of the human hand, or the operator use a glove with sensors, that acquire information that will be precessed to obtain an intelligent command and control of the robotic hands and arms.

\section{Methods That Are Used to Detect the Movements of the Hands and Arms of the Human Operator}

In 1998, researchers of Systems Lab at Southern Methodist University developed an exoskeleton system that operates with pneumatic action elements. The term exoskeleton (external skeleton) refers to a system of leverages, motors, actuators and sensors that is mounted on the hands and arms of the operator (this kind of systems have applications also in prosthetic and recuperatory medicine).

In the robotic system developed by Systems Lab, the exoskeleton is made of aluminum mobile elements having four joints each that detect the movement of the shoulder and elbow of the operator's arm. In figure 1 is visible the standpoint of the exoskeleton (behind the operator's shoulder); the aluminum elements may be adjusted depending on the physical characteristics of the operator, to fit on the right arm. In order to command the metal structure (which has large dimensions), the human operator commands the engines associated with each part of the system, through the movements of the fingers, arm and forearm.

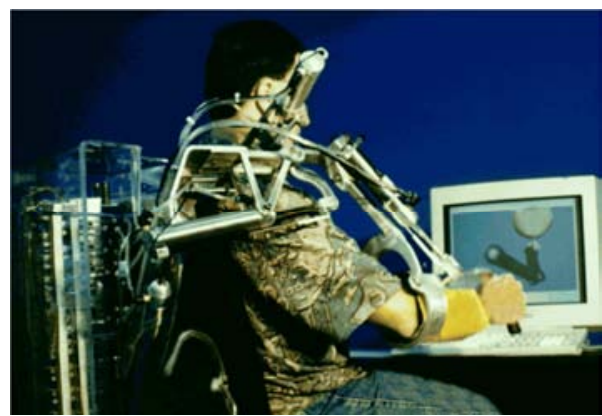

Figure 1. The exoskeleton robotic system realized by the researchers of Systems Lab at Southern Methodist University [3].

The robot named Mahru was developed by Korea Institute of Science and Technology (KIST) and Samsung Electronics uses the same method. The robot implements a large selection of complex movements for arms and legs, stored in its library. Figure 2 represents the exoskeleton that command the arms of the robot. It consists of leverages, sponsor and actuators and it detects and decomposes the movements of the operator - the corresponding movement is searched in the library of movements for the hand, arm and forearm.

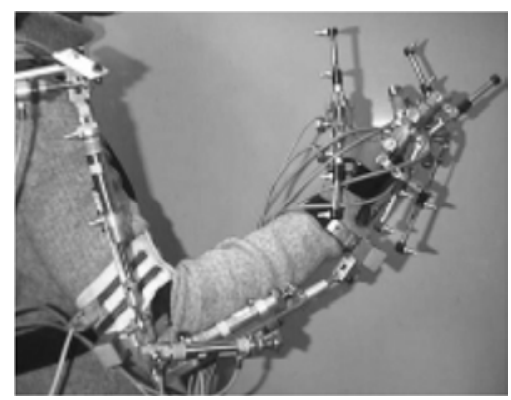

Figure 2. The exoskeleton system used to analyze the movement of the mobile elements of the human operator's arm. [4]. 
In both examples presented so far, the devices mounted of the joints of the hand and arm measure, memorize and process several movement parameters for different parts of the human arm, that are put in correspondence with the moving elements of the robotic arm. In order to replicate the human movements, the movement parameters are detected with high precision - and therefore the trajectory, speed and acceleration of each mobile segment are determined.

A different category of equipment developed for hand's movement detection are the "data gloves", also with variations for other parts of the body: locomotory interfaces, specialized devices for different type of feedback and interactive suits. Interactive data suits like the one in figure 3 are nowadays used not only in virtual reality computer games, but also in training simulator for divers, soldiers, parachute jumpers etc.

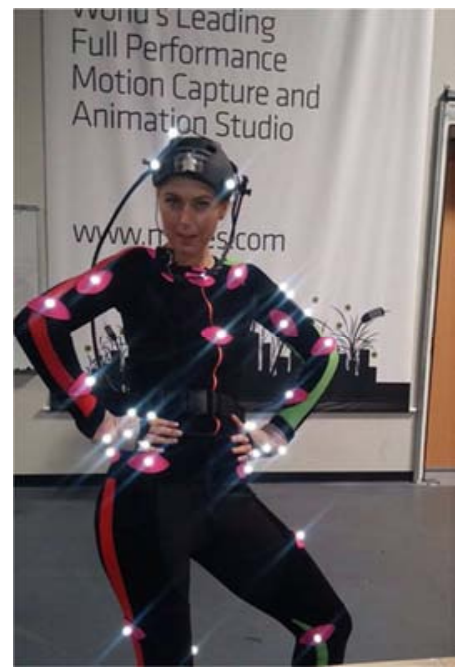

Figure 3. Costume used in virtual reality simulators [5].

Another real-time method for motion detection used to detect the motion of the human hand was developed by Elliptic Labs [6]. The method is based on an ultrasonic system with micro-electro-mechanic microphones and transmitters that detect and emit sonic waves in a specter of over $20 \mathrm{kHz}$. The acoustic waves are then processed and transformed in different commands for the computing circuits.

Data gloves (and systems that include data gloves) are the most used devices for real-time detection of the movements of the hands (including the movements of the arms) of the operators, since sun data gloves offer a complete solution for high precision detection of the movements of all joints.

Data gloves are mostly used for virtual reality applications. They have sensors inside the fingers of the gloves, tracking devices, accelerometers and force-feedback systems (that measure the resistance force when grasping objects and during different types of touch and interactions). The movements of the hand and finger are accurately detected and transmitted to the $\mathrm{PC}$, as input of specific applications. The accelerometers allows detection of two types of movements: dynamic and static.

The data gloves available on the market have different features and their performances depend on the number of sensors mounted on each finger, the sensors' resolution, and the force-feedback functions. They can detect complex movements, decomposed in up to 22 degrees; the accelerometers have up to 3 movement axis and is able to measure to rotation and elevation of the hand, in addition to the movements on the three axis. Cyber Glove is market leader for these products and produces various types of data gloves.

In certain applications that make use of mobile robots, the command and control of the movements of the mobile elements of the arms of the robot are based on computing systems of the type client-server. These systems use dedicated architectures and specialized software for the realtime processing of the information captured by the sensorial interfaces of the robot and its internal processing and memory blocks.

For instance, the mobile platform PowerBot and Power Cube Manipulator which is controlled with a client-server type of architecture. The architecture of the control block of this robot contains several micro controllers that give the control signals for orientation in a complex environment with high unpredictability and also control the interaction of the robot with this environment. This robot is manufactured by MobileRobots company that also developed software for implementation of high level control to avoid obstacles, for trajectory design, location, navigation and operation of the robotic arm. [7]

\section{Conceptual Modeling of Replication of the Movements of the Human Hand by Artificial Hands of the Robots}

The robotic systems that have been developed so far have a large variety of construction solutions, functional facilities and working conditions. Typical features refer to the number of fingers and their segmentation, number of segments of the arm, degrees of freedom, types of actions, working conditions like temperature and pressure etc.). However, no matter of the type of solution implemented, a robotic arm always will be designed as to be able to drive the effector (the robotic hand) in the required position, under the required angle (in order to realize a specific operation).

The interconnection of the human movements with the movements of the robotic arm implies to take into consideration the fact that the latter is actuated, usually, by more articulations (joints) than the human hand - typically, one for each degree of freedom. In order word, except for axial rotations of some segments (for instance, supine pronation of the forearm), the other double or triple rotations will be implemented with either two or three articulations for each human articulations (like those of the hand wrist or of the shoulder).

The detection of the movements of the human hand movements can be regarded as a technical and computing problem with different levels of complexity, depending on the number of degrees of freedom monitored and the precision 
needed in different applications. Starting with the monitoring of the movements of the human hand and arm, in direct correlation with the structure of the robotic arm that has to be controlled, adequate methods were conceived and selected for date processing and command of the action systems. [8]

In order to have a robotic arm and hand perform actions that are specific for humans, often the engineers have an empiric approach, specific for each type of articulated structure and its features (architecture of active elements, degrees of freedom). The movements of the human hand and hence the movements of the device that "copy" the human hands imply a certain number of articulated segments, each articulation having a limited number of degrees of freedom. The larger the number of segments and the larger the number of degrees of freedom, the greater will be the diversity of gestures and the higher the complexity of the movements therefore, the hand has more expressivity and more elaborate abilities. However, in the case of a robotic arm, the larger the number of articulations, the lower resistance (the robot will be fragile and vulnerable). The "/robot" is the whole systems that performs an action/function, and includes more blocks than the robotic arm.

There is a direction of research and development in robotics that is oriented toward the "reproduction" of the natural human arm and hand in models that copy more or less details of the latter. However, there are a lot of different solutions for robotic arms that are able to grasp and manipulate objects with simple to complex actions. In the scientific literature the main types of robotic hands and arms may have 2, 3 or 4 "fingers", each of them having 2 or 3 segments. Even those models try to "copy" some features of the human hand.

In order to replicate the movements of the human arm and forearm, we must take into consideration the degrees of freedom of the joints:

- shoulder - 3 degrees of freedom, of which 2 in vertical planes that are orthogonal (frontal and sagittal) and 1 for the rotation around the own axis;

- elbow - only 1 degree of freedom (flexion/extension)

- hand wrist - 3 degrees of freedom, of which 2 in vertical planes that are orthogonal and 1 for the rotation around the own axis (pronation/supination).

- For the hand, the articulations have the flexibility as follows

- thumb of the hand - the main joint (carpal-metacarpal) has two degree of freedom in vertical planes, while the metacarpal - phalanges and that between phalanges have only one degree of freedom

- other four fingers - the carpal-metacarpal articulations have only one degree of freedom (that allow small movements in order to modify the form of the palm), the metacarpal - phalanges articulations have two degrees of freedom each, and all articulations between phalanges have one degree of freedom.

Summing up, the arm and forearm have 7 degrees of freedom altogether, while the palm with the fingers have 24 degrees of freedom, of which 20 are explicit and large, the other 4 allowing only small movements to adjust the form of the palm to the form of the object handled and grasped.

In conclusion, a precise and complete mathematical representation of the position and movement of the arms should take into consideration all 27 explicit degrees of freedom, and corresponding parameters should be monitored in order to replicate the movement of the human hand.

\section{The Implementation of the Processing and Control Method for the Artificial Arm and Hand}

The data processing required for our application implies a large computing power, as real-time processing of a large quantity of data is needed. As classic computing models (i.e. with a central computing block) may not handle such processing, our solution is based on distributed and hierarchic computing architecture.

The block scheme of the computing architecture is represented in Figure 4. The processing tasks for the data acquired by sensors are locally processed, only results being transmitted to the superior level. The same hierarchy is implemented for the data processing of the robotic system moving parameters. The superior computing level will therefore not be overloaded by data and data transmission tasks. Therefore the scheme does not contain large capacity data buses, but a standard communication system micro controller - processor.

The detection of the movements of the human hand may be solved by different solutions, of different complexity, all aiming at replication of the movements of the human hand, but taking into consideration a different number of parameters, in order to obtain the required performances of precision and ability. The goal is to control the robotic hand through the movement of the operator's hand.

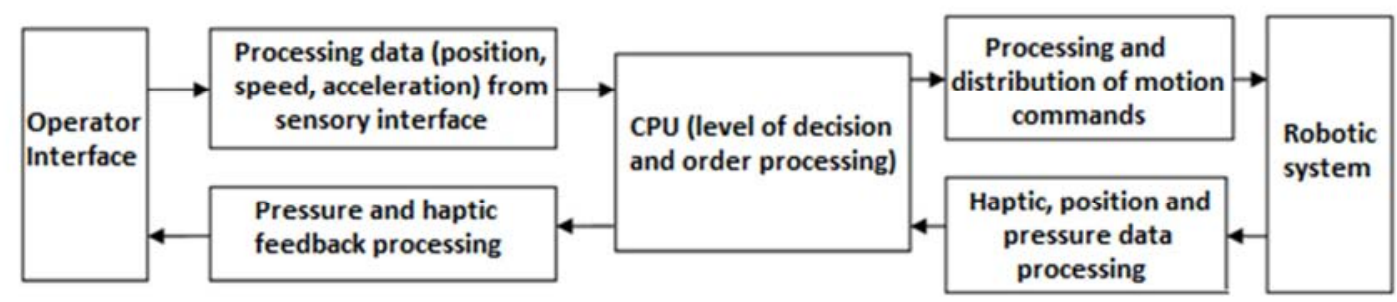

Figure 4. The hierarchic distributed processing scheme for the control of the robotic hand. 
To do this efficiently, a first level of computing is dedicated to high precision detection of the movements of the operator's hand (of which "segments of interest" may be accordingly defined, depending on application) and processing of the information, in order to command the robotic arm.

Figure 5 is a picture of the prototype of the robotic arm its mechanical structure. In the picture in figure 8 , all sensorial interfaces and processing elements are mounted on the prototype. As one can see, the model looks alike the human arm and hand (or a sketch of it), but there are significant differences regarding the cinematic and functionality.

The data processing is divided in two main sections:

- the command section of the data processing, in which the sensorial input from the hand of the operator is processed, transmitted and converted in appropriate commands;

- a local section, for local control of movements and stability, being responsible for, instance, for the adaptation of the actions to the form, weight and other characteristics of the manipulated objects.

In our hierarchic approach of the computing, the local section is subordinated to the central level of command [9] [10]. The effective action elements of the robotic hand and arm are controlled both by central commands and local controls. Specific feedback is transmitted to the operator in order to have an efficient remote control. In addition to the interfaces with the user and the robotic arm, several computation units are included in the architecture in order to may adjust the functionality to complex operations.

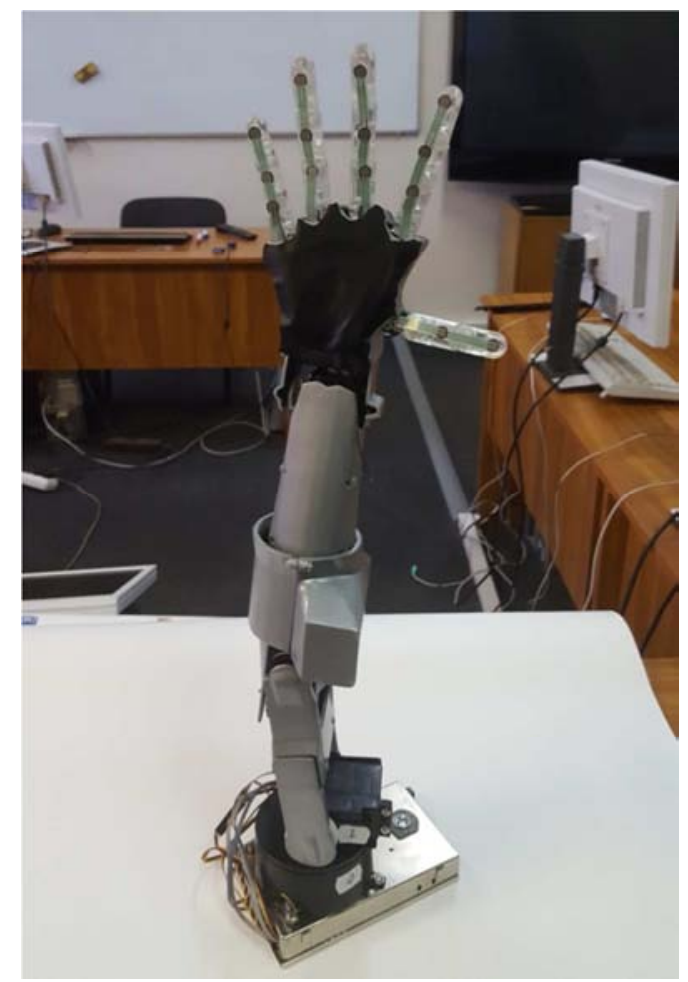

Figure 5. The mechanical structure of the prototype.
The design of the sensorial interface that monitors the movements of the operator took into consideration all mobile possibilities of the healthy human hand. The robotic arm was designed accordingly to the largest capacity and variety of movements, therefore it has 5 degrees of freedom. The robotic hand has an anthropomorphic structure (i.e. imitates the human hand, having 5 fingers with similar number of phalanges as the human fingers).

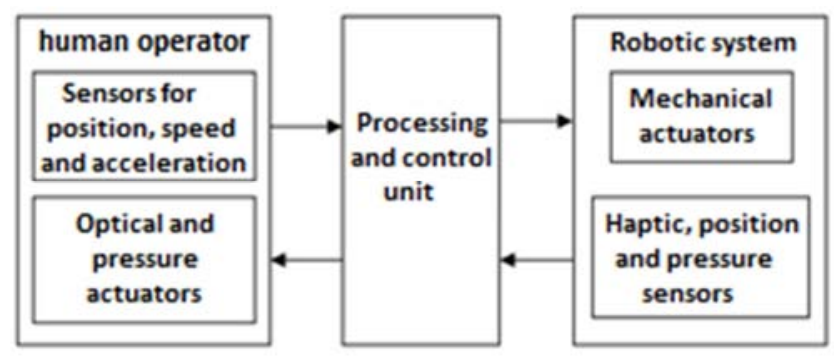

Figure 6. Interconnection of sensorial (data acquisition) systems and information processing blocks.

However, since the robotic arm/hand has more articulations than the human, the interconnection of the movements is not a direct replication of commands. The processing system converts the movement of the human hand into commands for the robotic hand. Except for the axial rotation of some segments (like the supine-pronation of the forearms, the other movements that imply double or triple rotations are realized by means of two/three articulations for each human articulation. Being more flexible than the human physical body, the articulations of the robotic arm allow rotations in the range of 30-180 degrees, depending on the position and utility of the articulation.

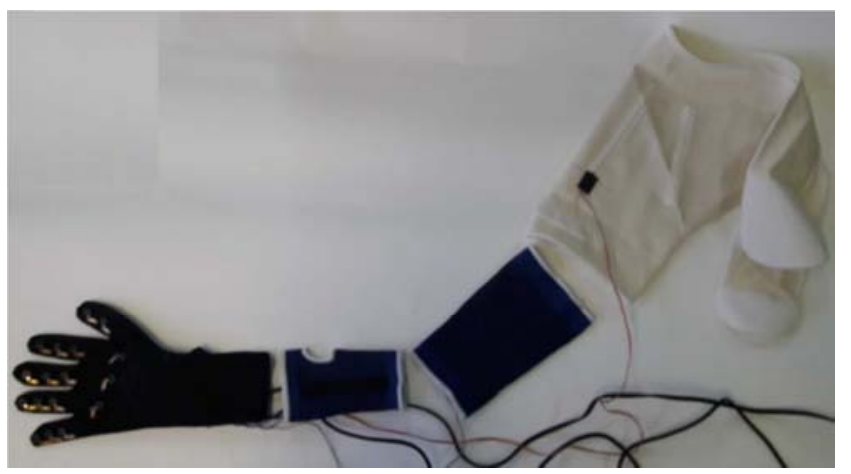

Figure 7. The sensorial interface that detects the movements of the joints and articulations when mounted on the human operator's arm.

The sensorial interface for the human arm (figure 7) consist of a data glove and a set of sensors for flexion, placed on the joints of the arm and hand, data acquisition systems and a processing unit. The result of the movement detection is transmitted to the central unit, equipped with the main microprocessor, which computes and gives the commands for the robotic arm.

The algorithms for data processing that generate the commands in order to replicate the human movements with 
the artificial structure were presented by the authors in [7]. The experiments realized so far had an empiric approach, testing the capacity of the robotic system to follow the movements of the human hand and the reproducibility of the actions performed.

The replication of the operator's movements was verified for several movements, at different speeds and acceleration, involving complex trajectories and rotations.

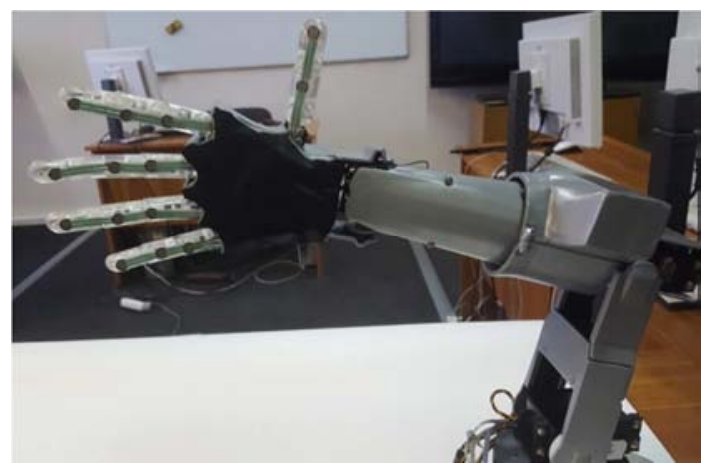

Figure 8. The anthropomorphic artificial hand with the "sensorial" haptic array implemented on the phalanges.

A set of basic movements were repeated for different parameters - the robotic arm repeated the movements. The measurements of the amplitude and trajectory of the movements showed small deviation (in the alignment) of the human/artificial arm. However, the robotic arm was able to grasp and lift objects and even to play a simple tune on a piano.

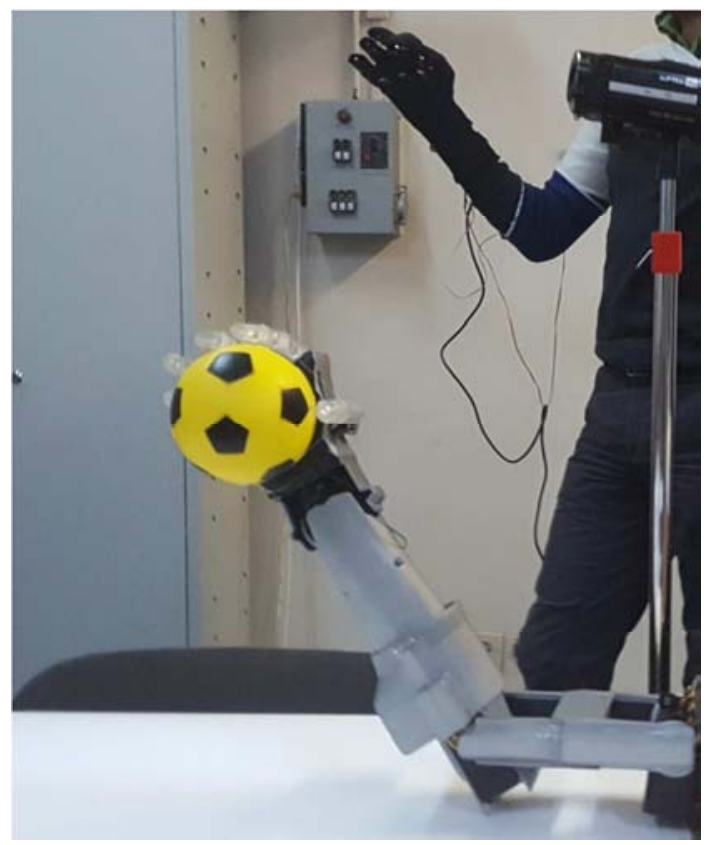

Figure 9. In this experiment, the operator tries to give the appropriate commands for grasping a small ball, based on the haptic feedback.

All mobile units have acted as designed, according to the corresponding segment of the operator arm and hand. The experiments certified the efficiency of the methods used for data acquisition and data processing.

\section{Conclusions}

This paper presents an innovative conception for teleoperation of a robotic arm: using data gloves and additional (similar) sensorial units, for the joints of the arm, as in virtual reality applications. The movements of the operator are duplicated by the robotic arm, although the structure is different in number of articulations and different motion components are involved.

The prototype has an anthropomorphic artificial hand (with 5 fingers) and was trained with basic operations of lifting, grasping and rotations [11]. The experiments had promising results, with the robotic arm replicating the human movements, although there are small alignment errors of the two. The limitations of the systems are due to the performance of the mechanical structure of the robotic anthropomorphic arm, including the fix and mobile segments and their joints.

For this phase of the project, the aim of the research was to solve the interconnection and processing issues, generating the commands for tele-operation - the applications will imply haptic feedback and $3 \mathrm{D}$ visual contact, therefore offering the possibility to correct the alignment with further feedback.

The experiments certified the efficiency of the architecture and algorithms and will be followed by further experiments with more complex and dynamic movements.

\section{Acknowledgement}

This work was supported by a grant of the Romanian National Authority for Scientific Research, Program for research - Space Technology and Advanced Research STAR, project number $82 / 2013$.

\section{References}

[1] Bicchi A, Kumar V. Robotic grasping and contact: a review. IEEE Intl Conf on Robotics and Automation Proceedings 2000; $1: 348-353$.

[2] Erika Nathalia Gama Melo, Oscar Fernando Aviles Sanchez, Darlo Amaya Hurtado, "Anthropomorphic robotic hands: a review", Ingeniería y Desarrollo, 32(2), 279-313. Retrieved September 28, 2015.

[3] Richer E, Hurmuzlu Y. A High Performance Pneumatic Force Actuator System: Part I-Nonlinear Mathematical Model. ASME. J. Dyn. Sys., Meas., Control. 1999; 122(3): 416-425. doi: $10.1115 / 1.1286336$.

[4] Sooyong Lee, Munsang Kim, Chong-Won Lee, "Human and Robot Integrated Teleoperation", 1998 IEEE International Conference on Systems, Man, and Cybernetics, Volume 2.

[5] Jack Bezants, Fancy a knock-up with Maria? US Open fans will be able to play against star in virtual reality as she shows off special suit, MailOnline, 19 August 2015, http://www.dailymail.co.uk/sport/tennis/article3203281/Maria-Sharapova-films-virtual-reality-tennisencounter-allow-supporters-Open-play-against-World-No2.html http://blog.mygreencorner.com/anapproach.pdf. 
[6] Matt Humrick, Elliptic Labs Demos Ultrasonic Touchless Gesturing Technology, Tom's Hardware, January 16, 2015, http://www.tomshardware.com/news/elliptic-labs-ultrasonicgestures, 28412.html.

[7] Lucian Milea, Monica Dascalu, Eduard Franti, Suzana Cismas, Doina Moraru, Florin Lazo, Elteto Zoltan. Detection and Tele-replication of Human Hand Motions by a Robotic Hand. American Journal of Aerospace Engineering. Vol. 2, No. 4, 2015, pp. 30-35. doi: 10.11648/j.ajae.20150204.11.

[8] Mihai Duguleana, An Approach to Solving Kinematics Models and Motion Planning for Manipulators with Mobile Base, Proceedings of the RAAD 2009 18th International Workshop on Robotics in Alpe-Adria-Danube Region May 25-27, 2009, Brasov, Romania.
[9] Voichița Dragomir, Breadth-First Search on a MapReduce One-Chip System, International Journal on Recent and Innovation Trends in Computing and Communication (IJRITCC), vol. 4, issue 4, April, pp 76-81, 2016.

[10] Voichița Maican, Minimum Spanning Tree Algorithm on MapReduce One-Chip Architecture, Romanian Journal of Information Science and Technology (ROMJIST), vol 18, no 2, pp. 127-143, 2015

[11] Pogărăsteanu M. E., Barbilian A. G., "Bionic hand exoprosthesis - Perspectives for the future in Romania", Journal of Medicine and Life, 2014 Oct-Dec; 7(4): 601-603. 